Article

\title{
Assessment of Non-Invasive Measurements of Oxygen Saturation and Heart Rate with an Apple Smartwatch: Comparison with a Standard Pulse Oximeter
}

\author{
Carmen Spaccarotella ${ }^{1,+}$, Alberto Polimeni ${ }^{2,+} \mathbb{D}$, Cinzia Mancuso ${ }^{2}$, Girolamo Pelaia ${ }^{3}$, Giovanni Esposito ${ }^{1}$ \\ and Ciro Indolfi ${ }^{2,4, *(1)}$
}

check for updates

Citation: Spaccarotella, C.; Polimeni, A.; Mancuso, C.; Pelaia, G.; Esposito,

G.; Indolfi, C. Assessment of Non-Invasive Measurements of Oxygen Saturation and Heart Rate with an Apple Smartwatch: Comparison with a Standard Pulse Oximeter. J. Clin. Med. 2022, 11, 1467. https://doi.org/10.3390/ jcm11061467

Academic Editor: Laurent Fauchier

Received: 30 January 2022

Accepted: 3 March 2022

Published: 8 March 2022

Publisher's Note: MDPI stays neutral with regard to jurisdictional claims in published maps and institutional affiliations.

Copyright: (C) 2022 by the authors. Licensee MDPI, Basel, Switzerland. This article is an open access article distributed under the terms and conditions of the Creative Commons Attribution (CC BY) license (https:// creativecommons.org/licenses/by/ $4.0 /)$.
1 Division of Cardiology, Department of Advanced Biomedical Science, Federico II University, 80138 Naples, Italy; carmenspaccarotella@gmail.com (C.S.); espogiov@unina.it (G.E.)

2 Center for Cardiovascular Research, Division of Cardiology, Department of Surgical and Medical Sciences, University Magna Graecia, 88100 Catanzaro, Italy; polimeni@unicz.it (A.P.); cinzia_mancuso@live.it (C.M.)

3 Department of Health Sciences, University Magna Graecia, 88100 Catanzaro, Italy; pelaia@unicz.it

4 Mediterranea Cardiocentro, 80138 Naples, Italy

* Correspondence: indolfi@unicz.it; Tel.: +39-0961-364-7668

+ These authors contributed equally to this work.

\begin{abstract}
The most commonly used method to assess peripheral oxygen saturation $\left(\mathrm{SpO}_{2}\right)$ in clinical practice is pulse oximetry. The smartwatch Apple Watch 6 was developed with a new sensor and an app that allows taking on-demand readings of blood oxygen and background readings, day and night. The present study aimed to assess the feasibility and agreement of the Apple Watch 6 compared with a standard $\mathrm{SpO}_{2}$ monitoring system to assess normal and pathological oxygen saturation. We recruited study participants with lung disease or cardiovascular disease and healthy subjects. A total of 265 subjects were screened for enrolment in this study. We observed a strong positive correlation between the smartwatch and the standard commercial device in the evaluation of $\mathrm{SpO}_{2}$ measurements $(\mathrm{r}=0.89, p<0.0001)$ and HR measurements $(\mathrm{r}=0.98, p<0.0001)$. A very good concordance was found between $\mathrm{SpO}_{2}$ (bias, -0.2289; SD, 1.66; lower limit, -3.49; and upper limit, 3.04) and HR (bias, -0.1052; SD, 2.93; lower limit, -5.84; and upper limit, 5.63) measured by the smartwatch in comparison with the standard commercial device using Bland-Altman analysis. We observed similar agreements and concordance even in the different subgroups. In conclusion, our study demonstrates that the wearable device used in the present study could be used to assess $\mathrm{SpO}_{2}$ in patients with cardiovascular or lung diseases and in healthy subjects.
\end{abstract}

Keywords: Apple Watch 6; COVID-19; $\mathrm{SpO}_{2}$

\section{Introduction}

The most widely used method to assess peripheral oxygen saturation $\left(\mathrm{SpO}_{2}\right)$ in clinical practice is pulse oximetry. The major advantage of this method is that it is non-invasive and has other benefits such as ease of use so that it can be used for multiple out-of-hospital measurements [1]. For these reasons, pulse oximetry is also often used in patients with COVID-19 to monitor peripheral oxygen saturation frequently [2]. Standard pulse oximetry measures the oxygen saturation in the blood by shining light at specific wavelengths through tissue. Deoxygenated and oxygenated hemoglobin absorb light at different wavelengths (660 $\mathrm{nm}$ and $940 \mathrm{~nm}$, respectively), and an algorithm processes the absorbed light in the pulse oximeter to display a saturation value. The smartwatch Apple Watch 6 was developed with a new sensor and an app that allow taking on-demand readings of blood oxygen and background readings, day and night [3]. Smartwatches are widespread and are increasingly being used for digital health information. For instance, the Apple Watch can reliably detect atrial fibrillation [4-6], and we previously showed the possibility of 
using this smartwatch to obtain multiple ECG leads [7] to detect ST-segment ECG changes. More recently, we and others demonstrated that it is possible to assess QTc measurements with the Apple Watch [8,9] and even Brugada syndrome ECG patterns [10]. Accordingly, the present study aimed to assess the feasibility and agreement of the Apple Watch 6 compared with a standard $\mathrm{SpO}_{2}$ monitoring system to assess normal and pathological oxygen saturation in a large cohort of patients with cardiovascular disease, patients with lung disease and healthy subjects.

\section{Materials and Methods}

We recruited study participants older than 18 years with lung disease or cardiovascular disease. A group of healthy subjects was also included for comparison. Exclusion criteria were as follows: (1) missing upper extremity, hand, or finger; (2) inability to wear a watch because of wrist circumference or edema of the arm, wrist, or hand; (3) clinical instability.

The Ethical Committee of the University Magna Graecia approved the study, and all subjects included in this study gave their written informed consent. The study conforms to the principles outlined in the Declaration of Helsinki and is independent from industry.

Research staff were trained to measure $\mathrm{SpO}_{2}$ and $\mathrm{HR}$ with a smartwatch and a standard pulse oximeter according to their manufacturers' guidelines. The $\mathrm{SpO}_{2}$ and $\mathrm{HR}$ measurements were obtained with the Apple Watch 6 (Apple Inc., Cupertino, CA, USA) and with a standard Nellcor Portable $\mathrm{SpO}_{2}$ Patient Monitoring System, PM10N (Medtronic, Minneapolis, MN, USA), placed on the index and middle fingers of the left hand (the same arm that was used for smartwatch measurement). The measurement accuracy of the latter system is \pm 2 digits in the range of values from $70 \%$ to $100 \%$ for saturation, and \pm 3 digits in the range of values from 20 to $250 \mathrm{bpm}$ for pulse rate [11]. The measurements with the Apple Watch 6 and the Nellcor system were taken within 1 minute of each other in order to ensure comparability between the two devices. All subjects followed the same measurement schedule, and all measurements were repeated two times and averaged. Since movement artifacts are factors that can affect the reliability of measurements when using the smartwatch, particular attention was paid to its correct placement. During the measurements, the arms were placed at rest on a table. The wrist and palm were placed face down on a flat surface and held steady. Particular attention was paid to ensuring that the Apple Watch fitted snugly against the wrist. The wristband was snug but comfortable, and the back of the Apple Watch touched the wrist. If the wrist bones prevented the watch from fitting snugly, it was moved along the arm to about 2.5 to $5 \mathrm{~cm}$ above the wrist [12].

The primary aim of the study was the head-to-head comparison of the measurements of $\mathrm{SpO}_{2}$ and HR by the smartwatch and the standard pulse oximeter. The secondary aim was the comparison of the measurements of $\mathrm{SpO}_{2}$ and $\mathrm{HR}$ between subgroups (lung disease, $\mathrm{CV}$ disease, healthy subjects).

Continuous variables are presented as mean \pm standard deviation. For the assessment of differences in metric outcome variables, we used paired $t$-tests, and in the case of binary variables we used chi-square tests. A one-way analysis of variance (ANOVA) was used to determine any statistically significant differences between the means of two or more independent (unrelated) groups. A $p$-value of $<0.05$ was considered statistically significant. Shapiro-Wilk tests were used to assess the normality of continuous variables. The correlation between the two technologies was assessed using linear regression and estimated with Pearson analysis for normally distributed data and Spearman analysis for nonparametric data [13]. A plot of the differences between techniques was created according to the method described by J.M. Bland and D.G. Altmann [14]. Statistical analyses were performed using MedCalc Statistical Software, version 14.8.1 (MedCalc Software, Ostend, Belgium), and GraphPad Prism, version 8.0.0 (GraphPad Software, San Diego, CA, USA). 


\section{Results}

A total of 265 subjects were screened for enrolment in this study. Three subjects were excluded from the study as it was impossible to obtain data from them, probably due to them having small wrists. In five subjects, it was not possible to assess oxygen saturation with the Apple Watch despite multiple attempts, for reasons that could not be detected. After screening, 257 subjects were included in the present study. Of these 257 subjects, 56 were healthy controls, 60 were patients with lung disease, and 141 were patients with cardiovascular disease. The study population is described in Table 1.

Table 1. Participant characteristics.

\begin{tabular}{|c|c|c|c|c|}
\hline & $\begin{array}{l}\text { Healthy Subjects } \\
\qquad(n=56)\end{array}$ & $\begin{array}{l}\text { Lung Disease } \\
\quad(n=60)\end{array}$ & $\begin{array}{l}\text { CV Disease } \\
(n=141)\end{array}$ & $p$ \\
\hline Age, $y \pm S D$ & $43.18 \pm 14.31$ & $71.23 \pm 10.44$ & $\begin{array}{c}69.21 \pm \\
11.53\end{array}$ & $<0.001$ \\
\hline Male, $n(\%)$ & $24(42.9)$ & $45(75)$ & $99(70.2)$ & $<0.001$ \\
\hline Weight, $n \pm \mathrm{SD}$ & $69.52 \pm 12.15$ & $77.59 \pm 17.35$ & $\begin{array}{c}76.22 \pm \\
15.08\end{array}$ & $<0.02$ \\
\hline Height, $n \pm \mathrm{SD}$ & $168.30 \pm 9.05$ & $166.47 \pm 7.37$ & $\begin{array}{c}165.51 \pm \\
7.93\end{array}$ & 0.25 \\
\hline $\mathrm{BMI}, n \pm \mathrm{SD}$ & $24.49 \pm 3.64$ & $27.90 \pm 5.41$ & $27.71 \pm 4.50$ & $<0.02$ \\
\hline Hypertension, $n(\%)$ & $10(17.9)$ & $52(86.7)$ & $126(89.4)$ & $<0.001$ \\
\hline Diabetes mellitus, $n(\%)$ & $4(7.1)$ & $21(35)$ & $47(33.3)$ & $<0.001$ \\
\hline Dyslipidemia, n (\%) & $7(12.5)$ & $29(48.3)$ & $123(87.2)$ & $<0.001$ \\
\hline ACS, $n(\%)$ & $0(0)$ & $1(1.7)$ & $50(35.5)$ & $<0.001$ \\
\hline CCS, $n(\%)$ & $0(0)$ & $9(15)$ & $64(45.4)$ & $<0.001$ \\
\hline Stroke/TIA, $n(\%)$ & $0(0)$ & $3(5)$ & $6(4.3)$ & $<0.001$ \\
\hline Smoke, $n(\%)$ & $15(26.8)$ & $6(10.0)$ & $22(15.6)$ & $<0.001$ \\
\hline COPD, $n(\%)$ & $0(0)$ & $35(58.3)$ & $16(11.3)$ & $<0.001$ \\
\hline OSAS, $n(\%)$ & $0(0)$ & $16(26.7)$ & $10(7.1)$ & $<0.001$ \\
\hline $\mathrm{O}_{2}$ therapy, $n(\%)$ & $0(0)$ & $24(40.0)$ & $18(12.8)$ & $<0.001$ \\
\hline Room temperature, $n \pm \mathrm{SD}$ & $21.79 \pm 1.32$ & $21.28 \pm 0.55$ & $21.32 \pm 0.91$ & 0.94 \\
\hline Body temperature, $n \pm \mathrm{SD}$ & $36.18 \pm 0.36$ & $36.20 \pm 0.38$ & $36.14 \pm 0.40$ & 0.98 \\
\hline Wrist circumference, $n \pm \mathrm{SD}$ & $16.15 \pm 1.38$ & $16.94 \pm 1.15$ & $17.03 \pm 1.39$ & 0.91 \\
\hline
\end{tabular}

TIA $=$ Transient Ischemic Attack; ACS = Acute Coronary Syndrome; CCS = Chronic Coronary Syndrome; BMI = Body Mass Index; OSAS = Obstructive Sleep Apnea Syndrome; COPD = Chronic Obstructive Pulmonary Disease.

Healthy subjects were younger than patients with lung or CV disease $(p<0.001)$ and had fewer risk factors. No differences were found regarding the technical features of measurements (room temperature, body temperature, wrist circumference; $p=\mathrm{NS}$ ). We observed strong positive correlations between the smartwatch and the standard commercial device in the evaluation of $\mathrm{SpO}_{2}$ measurements $(\mathrm{r}=0.89, p<0.0001)$ and HR measurements $(\mathrm{r}=0.98, p<0.0001)$ (Figure $1 \mathrm{a}, \mathrm{b})$.

A very good concordance was found between $\mathrm{SpO}_{2}$ measured by the smartwatch in comparison with the standard commercial device (bias, -0.2289 ; SD, 1.66; lower limit, -3.49; and upper limit, 3.04) using Bland-Altman analysis. Figure 2a shows the difference in $\%$ of $\mathrm{SpO}_{2}$ between the smartwatch and the standard commercial device plotted against the mean of the two readings. This difference was considered clinically nonsignificant. Similarly, an excellent agreement was found between HR measured by the smartwatch in comparison with the standard commercial device (bias, -0.1052 ; SD, 2.93; lower limit, -5.84; and upper limit, 5.63) using Bland-Altman analysis. Figure $2 \mathrm{~b}$ shows the difference in beats per minute in HR between the smartwatch and the standard commercial device plotted against the mean of the two readings. This difference was considered clinically nonsignificant. 


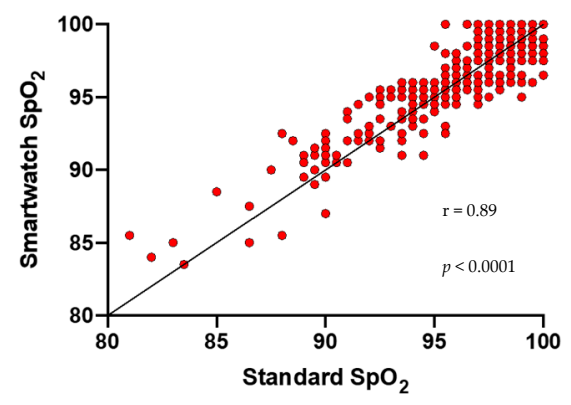

(a)

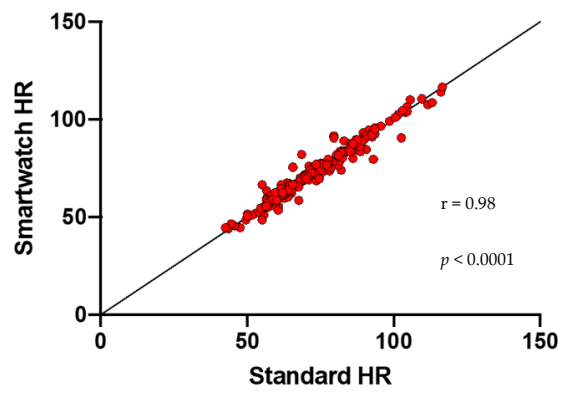

(b)

Figure 1. Correlation plots between the smartwatch and the standard commercial device. (a) Correlation between the smartwatch and the standard commercial device in the evaluation of $\mathrm{SpO}_{2}$ measurements $(\mathrm{r}=0.89)$. (b) Correlation between the smartwatch and the standard commercial device in the evaluation of HR measurements $(r=0.98)$.

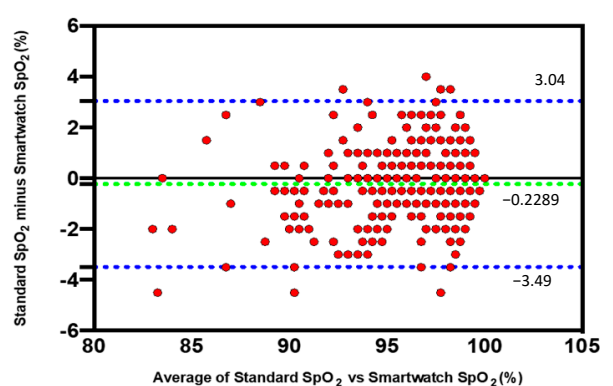

(a)

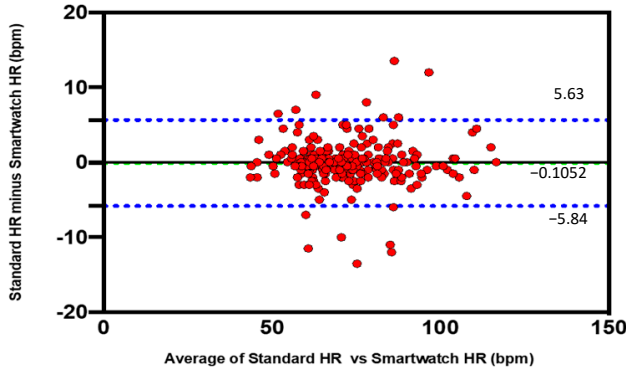

(b)

Figure 2. Concordance between $\mathrm{SpO}_{2}$ and $\mathrm{HR}$ measured by the smartwatch in comparison with the standard commercial device using Bland-Altman analysis. Bland-Altman plots indicate the level of agreement between the smartwatch and the standard commercial device. The dashed green line represents the bias (mean difference), and the dashed blue lines represents the upper and the lower limits of agreement. This difference is considered clinically nonsignificant. (a) Difference in \% of $\mathrm{SpO}_{2}$ between the smartwatch and the standard commercial device plotted against the mean of the two readings. (b) Difference in beats per minute in HR between the smartwatch and the standard commercial device plotted against the mean of the two readings.

Furthermore, based on the mean differences between the smartwatch and the standard commercial device, no statistically significant differences were found in both $\mathrm{SpO}_{2}$ and $\mathrm{HR}$ measurements ( $p=0.46$ and $p=0.93$, respectively) (Figure 3a,b).

We observed similar agreements and concordance between the standard commercial device and the smartwatch even in the different subgroups (lung disease, cardiovascular disease) for both parameters, $\mathrm{SpO}_{2}$ and $\mathrm{HR}$ (Figure S1). 


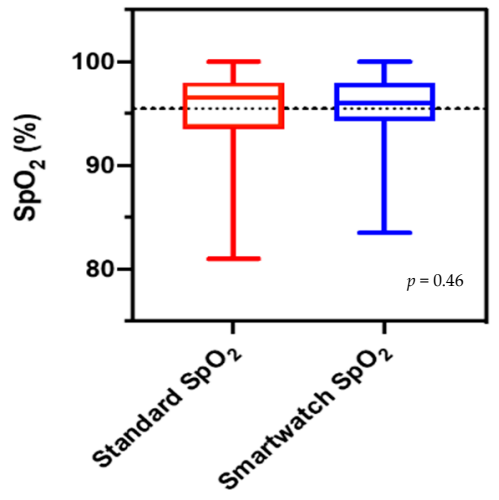

(a)

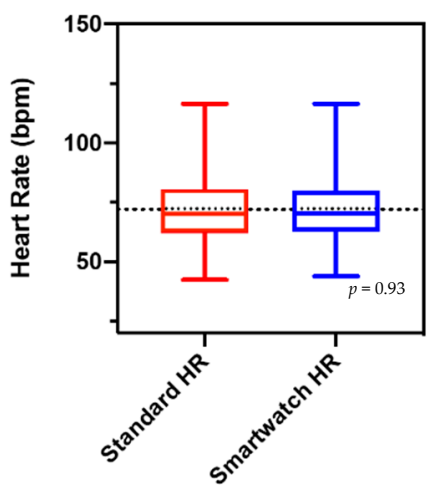

(b)

Figure 3. Boxplots of the mean differences between the smartwatch and the standard commercial device. (a) Mean difference in $\mathrm{SpO}_{2}$ between the smartwatch and the standard commercial device $(p=0.46)$ (b) Mean difference in HR between the smartwatch and the standard commercial device. $(p=0.93)$.

\section{Discussion}

The major result of the present study is that the measurements of $\mathrm{SpO}_{2}$ obtained using the Apple Watch are reliable compared to the standard pulse oximetry technique in patients with cardiovascular disease, lung disease, and healthy subjects. It has been estimated that the number of Apple Watches in use worldwide is about 100 million [15]. These smartwatches are used today in cardiology, especially for the accurate and reliable diagnosis of atrial fibrillation [4-6]. Furthermore, the possibility of measuring changes in the ST segment has recently been demonstrated in our laboratory for the first time [7]. In this study, the watch was placed in different body positions to obtain nine bipolar ECG tracings (corresponding to Einthoven leads I, II, and III). The multichannel smartwatch ECG reliably identified ST-segment changes in patients with acute coronary syndromes (NSTEMI and STEMI). Finally, we and others demonstrated that the smartwatches could be used in measuring the QT interval [8,9] and even Brugada syndrome [10]. As an additional feature, Apple developed the smartwatch Apple Watch 6 (Apple Inc, Cupertino, CA, USA) with a new sensor that consists of four LED clusters and four photodiodes. Incorporated into a completely redesigned crystal, this new sensor works in concert with the blood oxygen app to determine blood oxygen levels. Green, red, and infrared LEDs shine light onto the blood vessels in the wrist, and photodiodes measure the amount of light reflected. A recent study by Pipek et al. in outpatients with chronic obstructive pulmonary disease or interstitial lung diseases observed positive correlations between the Apple Watch device and commercial oximeters when evaluating heart rate measurements $(\mathrm{r}=0.995, p<0.001)$ and oximetry measurements $(\mathrm{r}=0.81, p<0.001)$ [16]. Our study is larger and improves on prior studies on this topic by including patients with cardiovascular disease. However, in contrast with the study by Pipek et al. [16], our study did not demonstrate differences in mean values of $\mathrm{SpO}_{2}$ measured with the Apple Watch compared to standard oximeters (Figure 3a). Therefore, our data did not show higher values with the Apple Watch compared to standard oximeters. Our data demonstrated a very good concordance between the $\mathrm{SpO}_{2}$ measured by the smartwatch compared with the standard commercial device (bias, -0.2289 ; SD, 1.66; lower limit, -3.49 ; and upper limit, 3.04). Therefore, the continuous monitoring of blood oxygen saturation with the wearable device assessed in the present study can be beneficial in various settings, both in patients with cardiovascular or lung diseases and in healthy subjects.

There are several limitations of the present report. In our study, even under ideal conditions, in a small percentage of cases (eight subjects) it was not possible to measure oxygen levels with the smartwatch. Skin perfusion, the anatomical variability of the wrist, 
and other reasons could be responsible. The data from our study were acquired in a wellcontrolled environment with a constant room temperature $\left(20 \pm 2{ }^{\circ} \mathrm{C}\right)$. Therefore, these results might not apply to different temperatures and environments-for example, in cold or hot temperatures. Another significant limitation is the lack of laboratory data as well as the fact that there were few subjects with saturation $<90 \%$ or with heart rate $>100 / \mathrm{min}$. The Apple Watch was used by an expert medical operator and the data accuracy might not apply to a broad population of users. Permanent or temporary changes to the skin, such as some tattoos, are another factor that can affect measurements. The ink used in some tattoos, as well as their design and saturation, can block light from the sensor, preventing the $\mathrm{O}_{2}$ levels app from taking measurements. The accuracy of pulse oximetry can be influenced by multiple factors, including perfusion and skin pigmentation [17]. In our population, however, all subjects were white and without tattoos in the skin area used for smartwatch use.

\section{Conclusions}

In conclusion, our study demonstrates that the wearable device used in the present study could be used to assess $\mathrm{SpO}_{2}$ in patients with cardiovascular or lung diseases and in healthy subjects.

Supplementary Materials: The following supporting information can be downloaded at: https:// www.mdpi.com/article/10.3390/jcm11061467/s1, Figure S1. Agreements and concordance between the standard commercial device and the smartwatch in the different subgroups. Table $\mathrm{S} 1 . \mathrm{SpO}_{2}$ Correlation between the smartwatch and the standard commercial device in different subgroups. Table S2. $\mathrm{SpO}_{2}$ Concordance between the smartwatch and the standard commercial device in difference subgroups. Table S3. HR Correlation between the smartwatch and the standard commercial device in different subgroups. Table S4. HR Concordance between the smartwatch and the standard commercial device in difference subgroups.

Author Contributions: Conceptualization, C.S. and C.I.; methodology, A.P.; software, A.P.; validation, C.S.; formal analysis, A.P.; investigation, C.M. and C.S.; resources, C.I.; data curation, C.M.; writing—original draft preparation, A.P.; writing—-review and editing, C.M. and C.I.; visualization, A.P.; supervision, G.E., G.P. and C.I.; project administration, C.I. All authors have read and agreed to the published version of the manuscript.

Funding: This research received no external funding.

Institutional Review Board Statement: The study was conducted in accordance with the Declaration of Helsinki and approved by the Ethical Committee of the University Magna Graecia.

Informed Consent Statement: Informed consent was obtained from all subjects involved in the study.

Data Availability Statement: The data underlying this article will be shared upon reasonable request to the corresponding author.

Conflicts of Interest: The authors declare no conflict of interest.

\section{References}

1. Chan, E.D.; Chan, M.M.; Chan, M.M. Pulse oximetry: Understanding its basic principles facilitates appreciation of its limitations. Respir. Med. 2013, 107, 789-799. [CrossRef] [PubMed]

2. NHS England. Specialty Guides for Patient Management during the Coronavirus Pandemic: Guidance for the Role and Use of Non-Invasive Respiratory Support in Adult Patients with Coronavirus (Confirmed or Suspected). Available online: https:/ / www.england.nhs.uk/coronavirus/wp-content/uploads/sites/52/2020/03/CLEARED_Specialty-guide_-NIVrespiratory-support-and-coronavirus-v2-26-March-003.pdf (accessed on 26 January 2022).

3. Apple.com. Available online: https://www.apple.com/Apple-Watch-Series-6/ (accessed on 26 January 2022).

4. Tison, G.; Sanchez, J.M.; Ballinger, B.; Singh, A.; Olgin, J.E.; Pletcher, M.J.; Vittinghoff, E.; Lee, E.S.; Fan, S.M.; Gladstone, R.A.; et al Passive Detection of Atrial Fibrillation Using a Commercially Available Smartwatch. JAMA Cardiol. 2018, 3, 409-416. [CrossRef] [PubMed]

5. Bumgarner, J.M.; Lambert, C.T.; Hussein, A.A.; Cantillon, D.J.; Baranowski, B.; Wolski, K.; Lindsay, B.D.; Wazni, O.M.; Tarakji, K.G. Smartwatch Algorithm for Automated Detection of Atrial Fibrillation. J. Am. Coll. Cardiol. 2018, 71, 2381-2388. [CrossRef] [PubMed] 
6. Perez, M.V.; Mahaffey, K.W.; Hedlin, H.; Rumsfeld, J.S.; Garcia, A.; Ferris, T.; Balasubramanian, V.; Russo, A.M.; Rajmane, A.; Cheung, L.; et al. Large-Scale Assessment of a Smartwatch to Identify Atrial Fibrillation. N. Engl. J. Med. 2019, 381, $1909-1917$. [CrossRef] [PubMed]

7. Spaccarotella, C.A.M.; Polimeni, A.; Migliarino, S.; Principe, E.; Curcio, A.; Mongiardo, A.; Sorrentino, S.; De Rosa, S.; Indolfi, C. Multichannel Electrocardiograms Obtained by a Smartwatch for the Diagnosis of ST-Segment Changes. JAMA Cardiol. 2020, 5, 1176-1180. [CrossRef] [PubMed]

8. Spaccarotella, C.A.M.; Migliarino, S.; Mongiardo, A.; Sabatino, J.; Santarpia, G.; De Rosa, S.; Curcio, A.; Indolfi, C. Measurement of the QT interval using the Apple Watch. Sci. Rep. 2021, 11, 10817. [CrossRef] [PubMed]

9. Strik, M.; Caillol, T.; Ramirez, F.D.; Abu-Alrub, S.; Marchand, H.; Welte, N.; Ritter, P.; Haïssaguerre, M.; Ploux, S.; Bordachar, P. Validating QT-Interval Measurement Using the Apple Watch ECG to Enable Remote Monitoring During the COVID-19 Pandemic. Circulation 2020, 142, 416-418. [CrossRef] [PubMed]

10. Spaccarotella, C.; Santarpia, G.; Curcio, A.; Indolfi, C. The smartwatch detects ECG abnormalities typical of Brugada syndrome. J. Cardiovasc. Med. 2021, 22, e24-e25. [CrossRef] [PubMed]

11. Medtronic.com. Available online: https://www.medtronic.com/content/dam/covidien/library/us/en/product/pulseoximetry/nellcor-portable-spo2-patient-monitoring-system-sell-sheet.pdf (accessed on 26 January 2022).

12. Modi, A.M.; Kiourkas, R.D.; Li, J.; Scott, J.B. Reliability of Smartphone Pulse Oximetry in Subjects at Risk for Hypoxemia. Respir Care 2021, 66, 384-390. [CrossRef] [PubMed]

13. Liu, J.; Tang, W.; Chen, G.; Lu, Y.; Feng, C.; Tu, X.M. Correlation and agreement: Overview and clarification of competing concepts and measures. Shanghai Arch. Psychiatry 2016, 28, 115-120. [PubMed]

14. Bland, J.M.; Altman, D.G. Measuring agreement in method comparison studies. Stat. Methods Med. Res. 1999, 8, 135-160. [CrossRef] [PubMed]

15. Macworld.co.uk. Available online: https://www.macworld.co.uk/news/how-many-apple-watches-sold-3801687/ (accessed on 26 January 2022).

16. Pipek, L.Z.; Nascimento, R.F.V.; Acencio, M.M.P.; Teixeira, L.R. Comparison of $\mathrm{SpO}_{2}$ and heart rate values on Apple Watch and conventional commercial oximeters devices in patients with lung disease. Sci. Rep. 2021, 11, 18901. [CrossRef] [PubMed]

17. Luks, A.M.; Swenson, E.R. Pulse Oximetry for Monitoring Patients with COVID-19 at Home. Potential Pitfalls and Practical Guidance. Ann. Am. Thorac. Soc. 2020, 17, 1040-1046. [CrossRef] [PubMed] 